\title{
Stochastic Non-Linear Pseudo-Random Sequence Generator
}

\author{
Mahmood A. Shamran * \\ Date of acceptance 30/6/2009
}

\begin{abstract}
:
Many of the key stream generators which are used in practice are LFSR-based in the sense that they produce the key stream according to a rule $y=C(L(x))$, where $L(x)$ denotes an internal linear bit stream, produced by small number of parallel linear feedback shift registers (LFSRs), and C denotes some nonlinear compression function. In this paper we combine between the output sequences from the linear feedback shift registers with the sequences out from non linear key generator to get the final very strong key sequence
\end{abstract}

Key words:Stochastic process, birth and death key generator (BDG), linear feedback shift register (LFSR), Pseudo- noise sequence, Auto-correlation function, Periodicity.

\section{Introduction:}

Random numbers (in some
sense) is important in many applications such as computer simulation, Monte Carlo integration, cryptography, randomized computation, ranging. In each case we need a sequence of numbers (or of bits) that "appears randomly", yet is repeatable. There is often a trade offin order to pass many tests that may be necessary to make the sequence generators very complex, making it hard to analyze the sequence with respect to the randomness measures. [1].

In 1999, we introduce a new type of random sequence key generator for stream cipher purpose, based on the stochastic process specially the birth and death process named "Birth and Death Key Generator",[BDG for short]. [2]

In this paper we introduce a new system for the same type, in fact we make a combination between the (BDG) and the linear feedback shift register. This system has a many strong points which make it very hard for attack methods.

\section{Preliminaries:}

1. Definition (Stochastic process): [3]

A stochastic process [S.P. for short $\left\{X_{t}\right\}$ is a collection $\left\{X_{t}: t \in I\right\}$ of random variables. Typically, $I$ is an interval in $\mathrm{R}$ (in such case we say that $\left\{X_{t}\right\}$ is a continuous time stochastic process), or a subset of $\{1,2, \ldots, n, \ldots\}$ (in such case we say that $\left\{X_{t}\right\}$ is a discrete time stochastic process. We also call $t \longleftrightarrow X_{t}(w)$ the sample function (or sample path) of the S.P.

\section{Definition (Linear feedback shift register): [1] \\ A linear feedback shift register} [LFSR for short] of length $\mathrm{m}$ over a ring $R$ with coefficients $q_{1}, q_{2}, \ldots, q_{m} \in R$ is a sequence generator whose state is an element $\mathrm{S}=\left(\mathrm{a}_{\mathrm{m}-1}, \mathrm{a}_{\mathrm{m}-2}, \ldots, \mathrm{a}_{0}\right)_{\in R^{m}}=\sum$, where $\sum \quad$ is the set of the input state And whose state change operation $r$ is given by

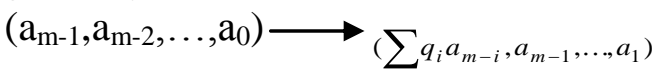

See fig(1) 


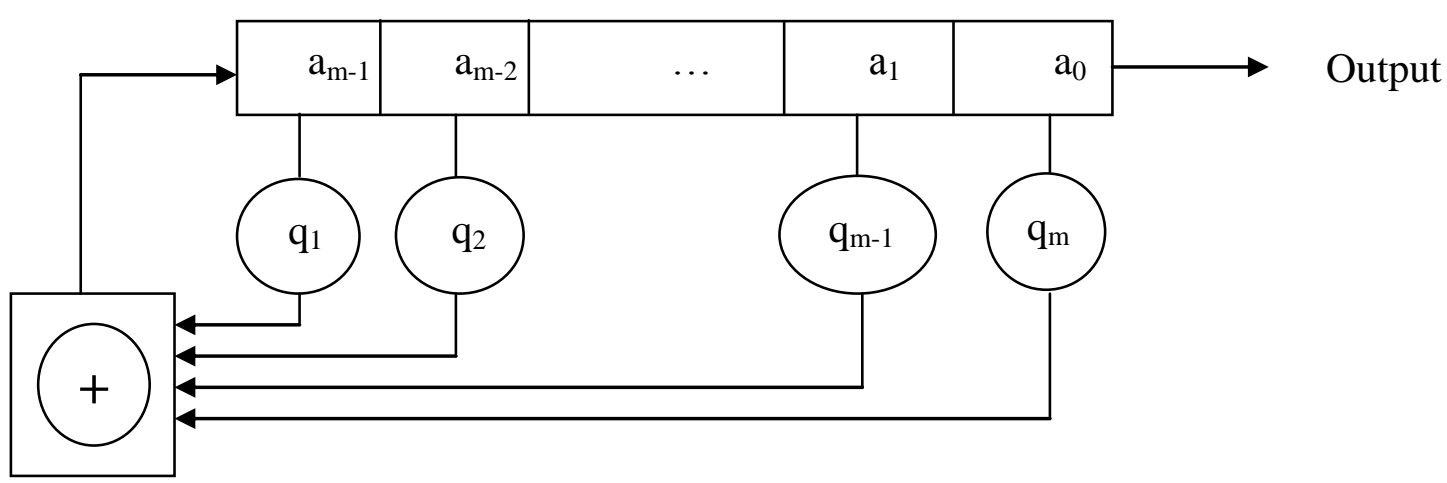

Figure(1) A linear feedback Shift register of length $\mathbf{m}$

\section{Definition (Linear feedback shift} register sequence): [4]

A linear feedback shift register sequence modulo $n$ of length $\mathrm{k}>0$ is a sequence $s_{1}, s_{2}, \ldots$ such that $s_{1}, s_{2}, \ldots, s_{k}$ are given and $s_{k+i} \equiv a_{1} s_{i}+a_{2} s_{i+1}+\ldots+a_{k} s_{k+i-1}(\bmod n)$ where $a_{1}, a_{2}, \ldots, a_{k}$ are given integers.

\section{Definition ( Period ): [1]} Let $A$ be a set and let $\mathrm{a}=\left(\mathrm{a}_{0}, \mathrm{a}_{1}, \mathrm{a}_{2}, \ldots\right)$ be a sequence of elements $a_{i} \in A$, the sequence $\mathrm{a}$ is periodic if there exists an integer $P>0$ such that $a_{i} \equiv a_{i+p}$ for all $\mathrm{i}=0,1,2, \ldots$ such that $P$ is called a period of the sequence a and the least $P$ is called period.

\section{Definition (Auto-correlation}

Function): [5]

The auto-correlation function is a way to quantize how random a sequence is and is defined by

$$
A C(k)=\frac{1}{p} \sum_{i=1}^{p} a_{i} \cdot a_{i+k}
$$

Where $p$ is the period of the sequence $\left\{a_{i}\right\}^{\infty}{ }_{i=1}$ and when $0<k<p, A C(k)$ is close to zero (meaning there is very little correlation of the sequence with itself) and $A C(k)=1 / 2$ when $k=0$, indicating that the number of 1 's is equal to the number of 0 s.

\section{Definition(Pseudo-noise Sequence):} [6]

$i>0$ Let $\left\{a_{i}\right\}_{i=1}^{\infty}$ be a binary sequence satisfying the Golomb's postulates:

1- The number of 1's in every period differ from the number of 0 's by at most one.

2- In every period, at least half of the runs must have length 1 , at least onefourth

length 2, etc., as long as the number of runs so indicated exceeds one. Moreover,

for each of these lengths, there must be (almost) equally many runs of 0 's and 1's.

3- $\left\{a_{i}\right\}^{\infty}{ }_{i=1}$ is a 2-level autocorrelation sequence. That is

$$
A C(k)=\left\{\begin{array}{c}
\boldsymbol{N} \text { if } k \equiv 0(\bmod N) \\
-1 \text { otherwise }
\end{array}\right.
$$

A binary sequence that satisfies the Golomb's postulates is called a pseudo-noise sequence or a pn-sequence.

\section{Remark:}

The linear feedback shift register sequence is pseudo-random sequence. 


\section{Definition (m-sequence): [1]}

A sequence $\mathrm{a}=\left\{a_{i}\right\}^{\infty}{ }_{i=1}$ is called $\mathrm{m}$-sequence (over a ring $\mathrm{R}$ ) of degree $r$ if it can be generated by a linear feedback shift register with length $r$, and if every nonzero block of length $r$ occurs exactly once in each period of a.

In other words, the sequence a is the output sequence of a LFSR that cycles through all possible nonzero states before it repeats.

\section{Birth and Death Key Generator (BDG) [2]}

Let $a$ and $b$ be two prime numbers such that a-1 and b-1 represent the order of cyclic group $Z_{a}, Z_{b}$ respectively, so that each of these groups has a generator elements.

$$
Y=\frac{\left(e^{-a}-b \cdot e^{-a / b}\right)+T\left(e^{-a / b}-e^{-a}\right)}{1-b}
$$

Where $T \in$ (set of the generator elements of $Z_{b}$ ), and $a, b$ represent the birth and death rate respectively, which are two generator elements in $Z_{a}, Z_{b}$.

The relation (1) represents the equation of straight line within the time interval $[1, b]$. And $P(x(t)=0)=\frac{d}{a}(1-Y)+0.5 Y$

Where $d \in($ set of the generator elements of $Z_{a}$ ).

If $P(x(t)=0) \leq 0.5 \quad$ then the generated bit is "1" and if $P(x(t)=0)>0.5 \quad$ Then the generated bit is " 0 ", repeat this process for each generator element of $Z_{a}$ with all the generator elements of $Z_{b}$ then we get the sequence of bits represent the output of the (BDG) key generator.

\section{Stochastic Non linear Key}

\section{generator :}

If we add the output sequence from the linear feedback shift register (LFSR) having a suitable length with delay by 1 bit to the output sequence from the (BDG). Then this new suggested key generator called Stochastic Non Linear Key Generator [SNG for short].

See fig (2).

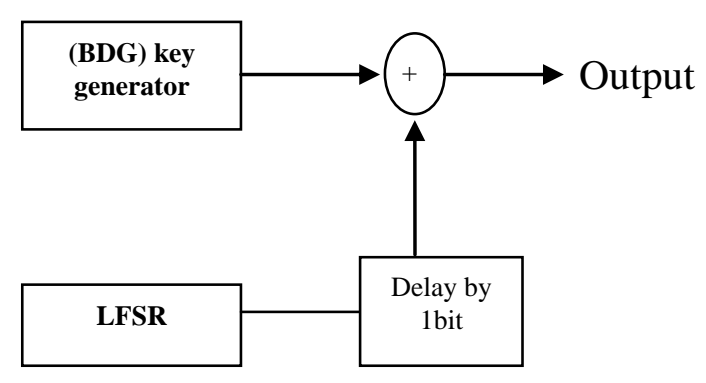

Figure (2) Stochastic Non linear key generator

\section{Main Results:}

The resulting sequences from the new suggested key generator (SNG) have the efficient criteria as shown in the sequent tables:

\section{Periodicity:}

Having a period is clearly a statistical defect that distinguishes a sequence from a random one. A cipher with a too small period is obviously easy to predict. The period must be large enough to ensure that is never repeated. It is usually done by using a building block that can be proven to have a large period, for instance a maximum-length LFSR.[6]

The period for the sequence output from this system is:

$\operatorname{per}($ SNGsequence $)=\mathrm{LCM}($ per $(\mathrm{LFSR})$, $\operatorname{per}(\mathrm{BDG}))$

\section{Complexity:}

The different complexities try to measure how hard a sequence is to produce. For a complexity measure to 
be practically useful for cryptographic purposes, two requirements have to be fulfilled.

- An efficient algorithm to calculate the complexity has to be known.

- The distribution of the complexity for random sequences has to be known If these requirements are fulfilled, the complexity measure can be used as a statistical

test. Complexity measures are often more interesting in cryptology than other statistical

17 tests, as some of them give methods to recreate the sequence using building blocks commonly used in stream ciphers. For the linear complexity, the distribution for a random sequence has been exactly calculated. It has been approximated for the maximum order complexity. Some complexity measures are only of theoretical interest because there is no efficient algorithm to calculate them.[6]

Then the linear complexity for (SNG) system is:
$\mathrm{LC}(\mathrm{SNG})=\mathrm{LC}(\mathrm{LFSR})+\mathrm{LC}(\mathrm{BDG})$

\section{Auto- Correlation:}

We can treatment this subject by using the delay by (1) bit to the sequence from the (LFSR) before adding it to the sequence from (BDG), this treatment deletes the strong correlation relationship between the output sequence with the sequence out from the (LFSR).

\section{Other Statistical tests:}

Regarding other tests such as run test, poker test, frequency test...etc. we test the output sequence from the (SNG)system to measure how hard a produce sequence. This results as shown in the tables below.

Table(1) shows The Periodicity, Linear Complicity[6] and Randomness (Frequency, Run and auto correlation) tests for some binary sequences which are generate from (SNG) with different prime numbers and LFSR length.

Table (1) efficiency criterions for (SNG) output results.

\begin{tabular}{|c|c|c|c|c|ccc|}
\hline LFSR length & $\boldsymbol{O P}$ & $\boldsymbol{O} T$ & $\boldsymbol{L}(\boldsymbol{S})$ & $\boldsymbol{L C}$ & \multicolumn{3}{|c|}{$\begin{array}{l}\text { Randomness } \\
\text { Run }\end{array}$ AC } \\
\hline \multirow{2}{*}{37} & 53 & 89 & 10000 & 4992 & $P$ & $P$ & F P P P P P P P P F \\
& 83 & 103 & 10000 & 4989 & $P$ & $P$ & $P$ P P P P P P P P P \\
\hline \multirow{2}{*}{43} & 211 & 163 & 50000 & 24985 & $P$ & $P$ & P P P P F F P P P F \\
& 59 & 61 & 50000 & 24966 & $P$ & $P$ & P P P P P P P P P P \\
\hline 501 & 457 & 367 & 100000 & 49986 & $P$ & $P$ & P P P P F P F P P P \\
\hline 601 & 83 & 691 & 250000 & 125012 & $P$ & $P$ & P P P P P P P P P P \\
\hline
\end{tabular}

Table(2) shows output results of various (SNG) system tested by CRYPT -X'98 using Periodicity, Linear Complicity, Frequency, Binary
Derivative, Change point, Sub block, Run and Sequence Complicity tests [6].

Table (2) tests results of SNG system for using XOR-CF.

\begin{tabular}{|c|c|c|c|c|c|c|c|c|c|}
\hline $\begin{array}{c}\text { LFSR } \\
\text { length }\end{array}$ & Primes & $\boldsymbol{L}(\boldsymbol{S})$ & $\boldsymbol{L C}$ & $\boldsymbol{F T}$ & $\boldsymbol{B D T}$ & $\boldsymbol{C P T}$ & $\boldsymbol{S B T}$ & $\boldsymbol{R} \boldsymbol{T}$ & $\boldsymbol{S C T}$ \\
\hline 103 & $\begin{array}{c}\text { OP 101 } \\
\text { OT 997 }\end{array}$ & 25000 & 11989 & $P$ & $P$ & $P$ & $P$ & $P$ & $P$ \\
\hline 523 & $\begin{array}{c}199 \\
1103\end{array}$ & 100000 & 49991 & $P$ & $P$ & $P$ & $P$ & $P$ & $P$ \\
\hline 613 & $\begin{array}{l}149 \\
509\end{array}$ & 500000 & 250103 & $P$ & $P$ & $P$ & $P$ & $P$ & $P$ \\
\hline
\end{tabular}




\section{Reference:}

1.Mark, G. and Andrew, K. 2008." Algebraic Shift Register Sequences": University of Toronto. pp 482 .

2.Mahmood, A. Sh. 1999.Thesis," Application of Markov Process To Generate Binary Sequences Which is Used in Stream Cipher Systems",ALMustansiriyah, University, Iraq.

3.Amir, D. 2008."Stochastic Process": Standford University. standford. pp131

4.Schneier, B. 1996. "Applied Cryptography: Protocols,
Algorithms, and Source Code in C", John Wiley \& Sons, New York, 2nd edition,pp1020.

5.Brock, B.T.2006."Linear Feedback Shift Registers and Cyclic Codes in SAGE": Mathematics Dept. USNA.pp221

6.MATTSSON， J. 2006.Thesis," Stream Cipher Design": Royal Institute of Technology School of Computer Science and Communication Stockholm, Sweden. مولا المتسلسلات شبه العثوائية اللاخطي التصادفي محمود عريبي شمران* | - | - (كلية العلوم للبنات/قسم الرياضيات/جامعة بغداد

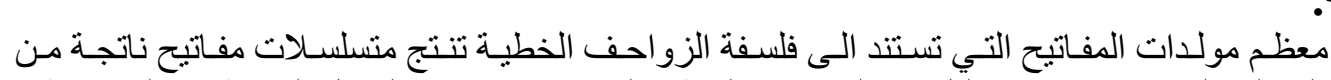

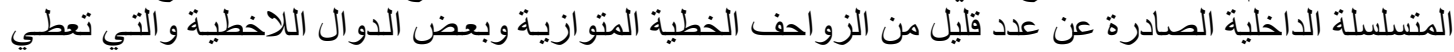

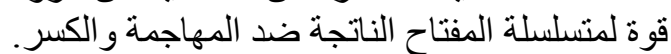

في هذا البحث تم المزج بين منسلسلة مفتاح من مولد لاخطي هو (Birth and Death Key Generator)

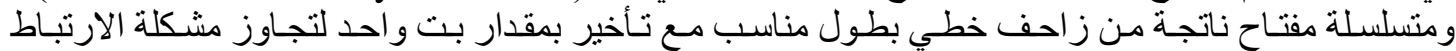

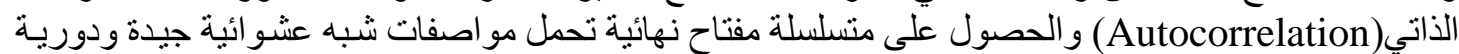

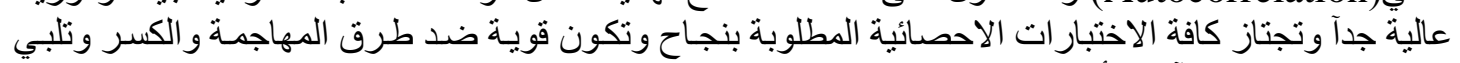
متطلبات الاستخدام الآمن لأغراض التثفير الانسيابي. 